\title{
Rapid Anatomical Plasticity of Horizontal Connections in the Developing Visual Cortex
}

\author{
Joshua T. Trachtenberg and Michael P. Stryker \\ Department of Physiology and W. M. Keck Center for Integrative Neuroscience, University of California, San Francisco, \\ San Francisco, California 94143-0444
}

Experience can dramatically alter the responses of cortical neurons. During a critical period in the development of visual cortex, these changes are extremely rapid, taking place in $2 \mathrm{~d}$ or less. Anatomical substrates of these changes have long been sought, primarily in alterations in the principal visual input from the thalamus, but the significant changes that have been found take 1 week. Recent results indicate that the initial physiological changes in the cortical circuit take place outside of the primary input layer. We now find that rapid plasticity of binocular re- sponses in the upper layers of cortex is mirrored by similarly rapid anatomical changes in the horizontal connections between ocular dominance columns in the upper layers, which reorganize within $2 \mathrm{~d}$.

Key words: V1; area 17; visual cortex; supragranular plasticity; corticocortical connections; pyramidal neurons; cat; striate cortex; strabismus; experience-dependent; horizontal collaterals
Experience refines the output of cortical circuits by introducing patterned activity that fine-tunes the strength of neuronal connections within and among cortical columns. During a critical period of early postnatal development, neuronal connections are extremely plastic. In the primary visual cortex (V1), decorrelating the activity from the two eyes, either by occluding the vision of one eye [monocular deprivation (MD)] or misaligning the optical axes (strabismus), changes cortical responses such that neurons come to respond to stimuli presented to one eye or the other but not to both (Hubel and Wiesel, 1963, 1965). This loss of cortical binocularity is ultimately mirrored by anatomical changes in the distribution of geniculocortical connections to the primary input layer IV (Shatz and Stryker, 1978).

At the height of the kitten critical period, plasticity of binocular responses is rapid. Either strabismus or MD induces a loss of cortical binocularity that saturates within $2 \mathrm{~d}$ (Olson and Freeman, 1975; Movshon and Dursteler, 1977; Van Sluyters and Levitt, 1980; Levitt and Van Sluyters, 1982). To date, no anatomical substrate for the rapid physiological plasticity has been found. Studies examining the rapidity of geniculocortical remodeling in monocularly deprived kittens have failed to demonstrate any change at $2 \mathrm{~d}$ after MD, with significant change requiring 4-7 d (Antonini and Stryker, 1993, 1996; Silver and Stryker, 1999, 2000).

Recent physiological results demonstrate that the initial site of cortical plasticity is at higher stages of cortical processing, outside of layer IV (Trachtenberg et al., 2000). These results are consistent with a number of studies that have examined experience-

Received Sept. 19, 2000; revised Feb. 5, 2001; accepted Feb. 22, 2001.

This research was supported by National Institutes of Health Grant R37EY02874. J.T.T. was the recipient of National Research Service Award EY06824. We are grateful to Prof. J. Winer for the use of his Neurolucida plotting system and to Drs. A. Antonini and L. E. White for counsel on anatomical procedures.

Correspondence should be addressed to either Prof. Michael P. Stryker, Department of Physiology, Box 0444, University of California, 513 Parnassus Avenue, Room S-762, San Francisco, CA 94143-0444, E-mail: stryker@phy.ucsf.edu; or Joshua Trachtenberg, Cold Spring Harbor Laboratories, 1 Bungtown Road, Marks Building, Cold Spring Harbor, NY 11724, E-mail: trachten@cshl.org.

Copyright $\odot 2001$ Society for Neuroscience 0270-6474/01/213476-07\$15.00/0 dependent cortical remodeling in the adult (Buonomano and Merzenich, 1998; Gilbert, 1998; Wallace and Fox, 1999; Feldman, 2000). Together, these data support a model in which functional reorganization is initiated in the upper layers. During the critical period, these changes can then guide the rearrangements ultimately seen in antecedent levels of sensory processing.

Possible substrates for upper layer plasticity include the vertical connections from layer IV to layer II/III (Feldman, 2000) and the long-range horizontal connections intrinsic to the upper layers that interconnect groups of neurons with similar functional properties (Gilbert and Wiesel, 1989; Malach et al., 1993; Bosking et al., 1997; Kisvárday et al., 1997). Our focus in this manuscript is on the plexus of long-range horizontal connections in layer II/III. The strength of the synapses formed by these connections can be rapidly potentiated or depressed (Hess et al., 1996; Rioult-Pedotti et al., 1998), and work in adult animals suggests that changes in this pathway are responsible for long-term functional changes in a number of cortical areas (Gilbert, 1998; Sanes and Donoghue, 2000).

If horizontal connections are a substrate of physiological plasticity in upper layers, one would expect their anatomical remodeling to take place at the same time as functional changes in the organization of the upper layers. Long-term strabismus produces an eye-specific segregation of the horizontal connections that would normally unite cells in the ocular dominance columns of the two eyes (Lowel and Singer, 1992). Here we examine the time course of horizontal connection remodeling after strabismus. We find that as little as $2 \mathrm{~d}$ of strabismic vision at the peak of the critical period produces a significant, if not saturated, loss of horizontal connections to opposite-eye ocular dominance domains, which enhances the eye specificity of these connections. These data provide additional evidence that rapid reorganizations of intracortical connections precedes and may subsequently direct the remodeling of thalamocortical connections.

\section{MATERIALS AND METHODS}

Nine kittens were studied: two normally sighted, five with $2 \mathrm{~d}$ of divergent strabismus induced between postnatal day 31 (P31) and P35, and two 
with long-term divergent strabismus of 1-2 week duration induced at P28 and P30. All procedures were approved by the University of California, San Francisco Committee on Animal Research.

Strabismus surgery. Kittens were anesthetized with $2-4 \%$ isoflurane in oxygen. A small incision was made in the nasal conjunctiva of one or both eyes. The tendonous insertion of the medial rectus muscle was caught and held using a small muscle hook. The muscle was resected near the myotendonous junction, and the muscle was allowed to retract. Chloramphenicol opthalmic ointment was instilled into the eye, and the animal was allowed to recover.

Optical imaging. Animals were surgically prepared for optical imaging and electrophysiological recordings as described previously (Issa et al., 1999). The cortical surface was illuminated using a tungsten-halogen light source and visualized through a tandem lens macroscope (two 50 $\mathrm{mm}$ lenses; $6 \times 8 \mathrm{~mm}$ total image area) attached to a CCD camera (ORA 2001; Optical Imaging Inc., Germantown, NY). Images of the pial vasculature were captured using a green $(546 \pm 10 \mathrm{~nm})$ interference filter and intrinsic signal responses to visual stimulation with a red $(610 \pm 10$ $\mathrm{nm}$ ) interference filter. Visual stimuli were displayed on a 21 inch monitor placed $40 \mathrm{~cm}$ from the animal. Stimuli consisted of drifting square wave gratings (spatial frequency, 0.2 cycles $/{ }^{\circ}$; temporal frequency, 2 cycles/sec; reversal of motion direction every $2 \mathrm{sec}$ ) of orientation $0^{\circ}$, $45^{\circ}, 90^{\circ}$, and $135^{\circ}$ with respect to horizontal. For each condition, 10 frames of $600 \mathrm{msec}$ duration [CCD binning set to $1 ; 1$ pixel $=(22.5 \mu \mathrm{m})^{2}$ ] were acquired. Ten conditions ( 4 orientations $\times 2$ eyes +2 blanks) repeated 16 times comprised one run. Four to six runs were analyzed for each experiment. Ocular dominance ratio maps were derived by dividing the sum of responses of one eye to all orientations by the responses of the other eye. Images were analyzed using commercial (ORA 2000) and custom software written in the Interactive Data Language (Research Systems, Boulder, CO).

Targeted tracer injections. Biocytin and wheat germ agglutinin-apoHRP-gold (WAHG) (Basbaum and Menterey, 1986) (generously supplied by J. Winer, University of California, Berkeley, CA) were injected extracellularly into regions of the cortex targeted on the ocular dominance maps. Injection sites were selected on the ocular dominance ratio maps as regions of strong ocular dominance. Selected sites were located using blood vessels on the cortical surface that were visible in the corresponding image of the pial vasculature. Biocytin was iontophoretically injected with a glass micropipette with a tip diameter of $8-12 \mu \mathrm{m}$ containing 3\% biocytin (Sigma, St. Louis, MO) in saline using pulsed current ( $7 \mathrm{sec}$ on, $7 \mathrm{sec}$ off) of $3 \mu \mathrm{A}$ for $10-20 \mathrm{~min}$. In some cases, the ocular dominance and orientation tuning of cells at the injection site was confirmed by recording multiunit activity through the injection micropipette. Typically, biocytin injections were made at the depth of 300-400 $\mu \mathrm{m}$. Twenty to $30 \mathrm{nl}$ of WAHG was pressure injected (Nanoject; Drummond Scientific, Broomall, PA) through a thick-walled glass micropipette with a tip diameter of $20-30 \mu \mathrm{m}$ advanced $250-400 \mu \mathrm{m}$ below the pial surface. After the tracer injection(s), the cortex was covered with low melting-point agarose ( $3 \%$ in saline), and the tracer was allowed to transport for 20-24 hr. During this time, the animal remained paralyzed and anesthetized. Rectal temperature, peak expiratory $\mathrm{CO}_{2}$, expiratory pressure, and electrocardiogram were continuously monitored. Adjustments in the respiratory rate and volume were made to maintain the peak $\mathrm{CO}_{2}$ between 2.5 and $4.5 \%$. Level of anesthesia was determined by monitoring the heart rate and peak $\mathrm{CO}_{2}$.

Tissue processing. After $20-24 \mathrm{hr}$ of transport time, the animal was deeply anesthetized with Nembutal $(10 \mathrm{mg}$, i.v. $)$ and transcardially perfused with $0.9 \%$ saline, followed by $5 \%$ formalin in acetate buffer, $\mathrm{pH}$ 6.5 , followed by $2.5 \%$ formalin- $-1.5 \%$ glutaraldehyde in borate buffer, $\mathrm{pH}$ 8.5. Tissue was cryoprotected in $30 \%$ sucrose before sectioning. For each hemisphere, $40-\mu \mathrm{m}$-thick sections were cut tangentially to the cortical surface. Special care was taken to retain the first few sections containing the pial vasculature, which was essential for later alignment with the optical maps. Biocytin labeling was visualized using a standard avidinbiotin (Vector Laboratories, Burlingame, CA) complex reaction and diaminobenzidine with nickel and cobalt intensification. Between 8 and 32 neurons were labeled in each section used in this analysis. WAHG labeling was visualized after silver intensification (Amersham Pharmacia Biotech, Arlington Heights, IL). This reaction produced a dense precipitate in a 500- to $700-\mu \mathrm{m}$-diameter region surrounding the injection site that is attributable to silver grains deposited early in the reaction serving as nucleation sites as the reaction proceeds. In sections that had not been silver-intensified, the injection site (and the effective transport site; Ruthazer and Stryker, 1996) was restricted to an area of between 100 and
$200 \mu \mathrm{m}$, much smaller than the region of dark precipitate present after silver intensification and much smaller than the diameter of a single ocular dominance column ( $\sim 450 \mu \mathrm{m}$ in the kitten).

Bouton-cell body plotting and alignment. For each tissue section, the blood vessels (superficial blood vessels in the first one to two sections; radial profiles in the remaining sections) and biocytin-labeled boutons and/or WAHG-labeled cell bodies were plotted using Neurolucida software (MicroBrightField, Colchester, VT). Biocytin-labeled boutons were visualized using bright-field illumination and plotted at $400 \times$ magnification $(40 \times$ air objective and $10 \times$ eye piece). In sections with high densities of labeled boutons, a grid composed of $50 \times 50 \mu \mathrm{m}$ boxes was overlaid on the section. All boutons in every third box were plotted. This anti-aliasing sampling method provided an accurate measure of the bouton distribution across ocular dominance domains. WAHG-labeled cell bodies were visualized using dark-field illumination and plotted at $100 \times(10 \times$ air objective and $10 \times$ eye piece $)$. The data from each section were stored as a series of $x, y$ coordinates. Alignment of the data with the optical maps was accomplished using custom software (J. T. Trachtenberg). The first stage was to align the plot of the superficial blood vessels taken from the first and second sections with the video image of the superficial blood vessels, which was taken from exactly the same region of V1 that was imaged for intrinsic signals. Global scaling, rotation, and translations were applied to the $x, y$ coordinates to render the best alignment. Deeper sections were aligned with the superficial sections using coevident profiles of radial blood vessels. Bouton and cell body plots were therefore aligned with the optical maps solely on the basis of their relationship to the pattern of blood vessels.

Data analysis. The selectivity of labeled cells and boutons for ocular dominance was evaluated by two different procedures. At a coarse level, and for comparison with earlier reports, the convex region containing all of the labeled cells or boutons in each hemisphere was divided into two zones: one dominated by the left eye and the other by the right eye, on the basis of visual responses measured from the intrinsic signal images. For a finer analysis of the pattern of connections in relation to visual responses, the optical maps from each hemisphere were divided into 10 ocular dominance zones of equal area, with the extreme zones (1 and 10) responding most strongly to one or the other eye and with a graded mixing of responses to the two eyes in the intermediate zones. The region measured in each hemisphere was either the convex region containing all of the labeled cells or boutons excluding a circle of radius 1 $\mathrm{mm}$ surrounding the injection site (referred to as long-range connections) or the annular region between 0.5 and $1 \mathrm{~mm}$ from the injection site (referred to as short-range connections). Within each of these regions, we measured the fraction of the area occupied by each of the 10 ocular dominance zones. We then calculated the enrichment or impoverishment of labeled cells or boutons within each ocular dominance zone by dividing the fraction of the total numbers of labeled cells or boutons in each zone by the fraction of area occupied by that zone, measured as described above. For uniform, random connections, the enrichment calculated in this manner would be 1 (that is, no enrichment or impoverishment). If all of the connections were made exclusively with the $10 \%$ of cortex having the most extreme same-eye ocular dominance, the enrichment would be 10 . Measured values of enrichment ranged between $\sim 6$ (sixfold enrichment) and 0.1 (90\% impoverishment). It should be noted that the relationship between physiological ocular dominance domains and the labeled cells or boutons was not known until after the alignment was performed, well after all of the cells and boutons had been plotted. This sequence ensured that all of the data were obtained using a blind procedure.

\section{RESULTS}

Connections among neurons in the upper layers of cortex were measured in two ways: (1) by finding the locations of the cell bodies that sent axons to a deposit of retrograde label, and (2) by finding the locations of the synaptic boutons made by cells labeled with an anterograde tracer. In each case, the label was targeted to a point in visual cortex strongly dominated by one eye as determined by optical imaging. If upper layer corticocortical connections between regions serving the two eyes are a substrate of rapid plasticity, they should be expected to change quickly when visual experience is manipulated, as do the binocular responses of neurons. The experiment below measured the time course of the anatomical remodeling of layer II/III horizontal connections dur- 

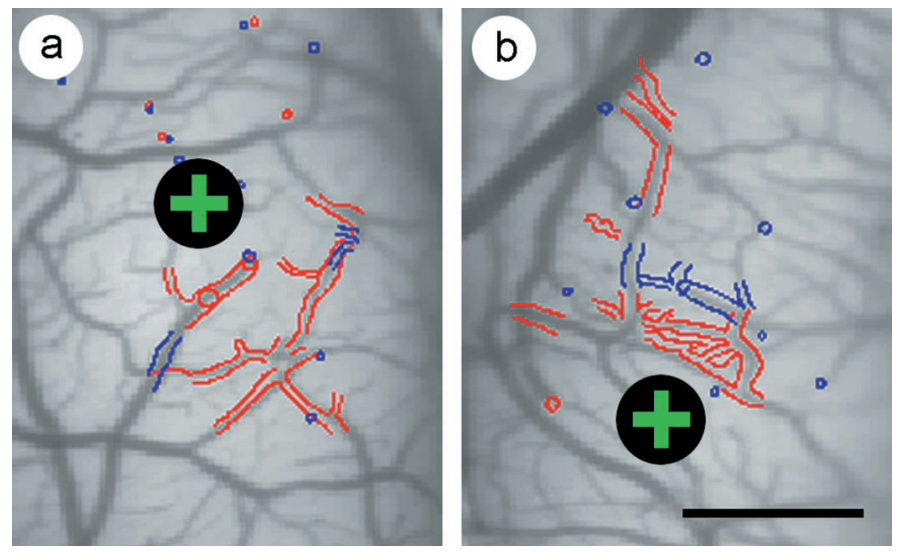

Figure 1. Alignment of anatomical sections to optical maps. The alignment of the first two anatomical sections (red and blue, respectively) with an image of the superficial blood vessels is shown for a normally sighted kitten $(a)$ and for a kitten exposed to $2 \mathrm{~d}$ of strabismus $(b)$. In both $a$ and $b$, the injection site of the anatomical tracer is marked with a black circle enclosing a green + . Computer-assisted drawings of the anatomical sections were independently scaled, rotated, and translated to achieve the best alignment with the vascular image. Outlines of the pial vasculature were used to align the first two sections with the vascular image. Coevident profiles of radial blood vessels were used to align deeper sections with more superficial sections. In this manner, the distribution of retrogradely labeled cells and anterogradely labeled boutons were brought into register with the optical maps of ocular dominance. Scale bar, $1 \mathrm{~mm}$.

ing the critical period for visual cortical plasticity and compared it with the known physiology.

\section{Retrograde and anterograde tracer injections}

To examine the time course of the anatomical remodeling of layer II/III horizontal connections, we studied the changes produced by misalignment of the visual axes for $2 \mathrm{~d}$ or $1-2$ weeks in nine cats at the peak of the critical period of plasticity. We used intrinsic signal optical imaging to map the distribution of responses to the two eyes in primary visual cortex and then targeted injections of retrograde (WAHG) and/or anterograde (biocytin) tracers to specific ocular dominance domains in normally sighted and strabismic kittens. Details of the injection protocol, plotting, and alignment of each section with intrinsic signal optical maps and subsequent analysis of the distribution of plotted boutons-cell bodies are provided in Materials and Methods. Examples of the initial steps in the alignment of the anatomical sections with the intrinsic signal optical maps are shown in Figure 1.

\section{The distribution of WAHG-labeled cell bodies}

To ensure that injections in control and strabismic kittens were made in similar functional domains relative to the map of ocular dominance, the positions of injection centers relative to peaks of ocular dominance were measured (ocular dominance peaks were determined as by Crair et al., 1997a). In control kittens, WAHG injections were centered $135 \pm 43 \mu \mathrm{m}$ from ocular dominance peaks. For animals made strabismic for 2 and 7-14 d, these distances were $134 \pm 16$ and $119 \pm 70 \mu \mathrm{m}$, respectively, which were not significantly different from control injections $(p>0.90$ for all comparisons). All injections, therefore, were placed well within monocular core zones.

In normally sighted (4 hemispheres, 10 sections, 5974 cells), $2 \mathrm{~d}$ strabismic ( 2 hemispheres, 5 sections, 2214 cells), and 7-14 d strabismic ( 2 hemispheres, 4 sections, 1597 cells) animals, retrogradely labeled cells had a clustered appearance. Examples of anatomical sections and the corresponding computer-assisted $x, y$ plots of cells from these sections from normally sighted and $2 \mathrm{~d}$ strabismic animals are presented in Figure $2 a-d$. These data are consistent with the known selectivity for connections with cortical columns of like orientation preference (Gilbert and Wiesel, 1989; Malach et al., 1993; Bosking et al., 1997).

The eye specificity in the pattern of horizontal connections in normally sighted animals differed dramatically from that in strabismic animals. Figure $2 e-g$ presents these differences quantitatively using two separate methods. In the first, the ocular dominance maps were divided into two ocular dominance domains, allowing a direct comparison with data presented by Lowel and Singer (1992). In the second, ocular dominance maps were divided into 10 ocular dominance domains, enabling a finer examination of the normal and strabismus-induced selectivity of horizontal connections relative to the physiological balance between the inputs of the two eyes. A more detailed description of this analysis is given in Materials and Methods. Results of these analyses on retrogradely labeled neurons are provided in Figure $2 e-g$.

Dividing the retrogradely labeled cells coarsely into two ocular dominance domains, we observed little bias in the eye specificity of horizontal connections in normally sighted animals. A pronounced bias in favor of connections with same-eye domains was seen as early as $2 \mathrm{~d}$ after the induction of strabismus $(p<0.05$; two-tailed $t$ test). This bias was further enhanced by prolonging the strabismus to $1-2$ weeks ( $p<0.05$; two-tailed $t$ test).

The finer-scaled analysis, in which ocular dominance maps were divided into 10 equal areas, revealed that 1-2 weeks of strabismus causes a large change in the optical responses at the extremes of ocular dominance (Fig. $2 f$ ). Concomitant with this change was a progressive remodeling of horizontal connections resulting in an essentially bimodal distribution of retrogradely labeled cells, as shown in Figure $2 g$. In control animals, there are $\sim 40 \%$ more labeled cells per unit area at the positions of extreme dominance by the same eye, with corresponding impoverishment of labeled cells at the peaks of opposite-eye dominance. However, in the $75 \%$ of cortex that is most binocular, we found little or no evidence for eye specificity of the connections. In contrast, in animals made strabismic for $2 \mathrm{~d}$, the most binocular zones, representing only $30 \%$ of the cortex, lacked eye specificity in their connections. In these animals, there was more than twofold enrichment in the numbers of labeled cells in the domain most strongly dominated by the same eye, with corresponding impoverishment of cells in opposite-eye domains. Connections after 1-2 weeks of strabismus presented a picture of further segregation and essentially complete loss of the binocular zone. Here the distribution of labeled cells was essentially bimodal, with similar enrichment in labeled cells throughout the $50 \%$ of the cortex dominated by the same eye and similar impoverishment in the half of the cortex dominated by the other eye.

\section{The distribution of biocytin-labeled boutons}

A higher resolution view of horizontal connections was obtained from plotting the positions of presynaptic boutons on the axonal collaterals of cells labeled with biocytin. As with the WAGH injections, care was taken to target the biocytin injections to monocular core zones. Biocytin injections in control, $2 \mathrm{~d}$ strabismic, and 7-14 d strabismic animals were centered $140 \pm 31,102 \pm$ 56 , and $121 \pm 31 \mu \mathrm{m}$, respectively, from targeted ocular dominance peaks. These distances are not significantly different from one another ( $p>0.50$ for all comparisons). 

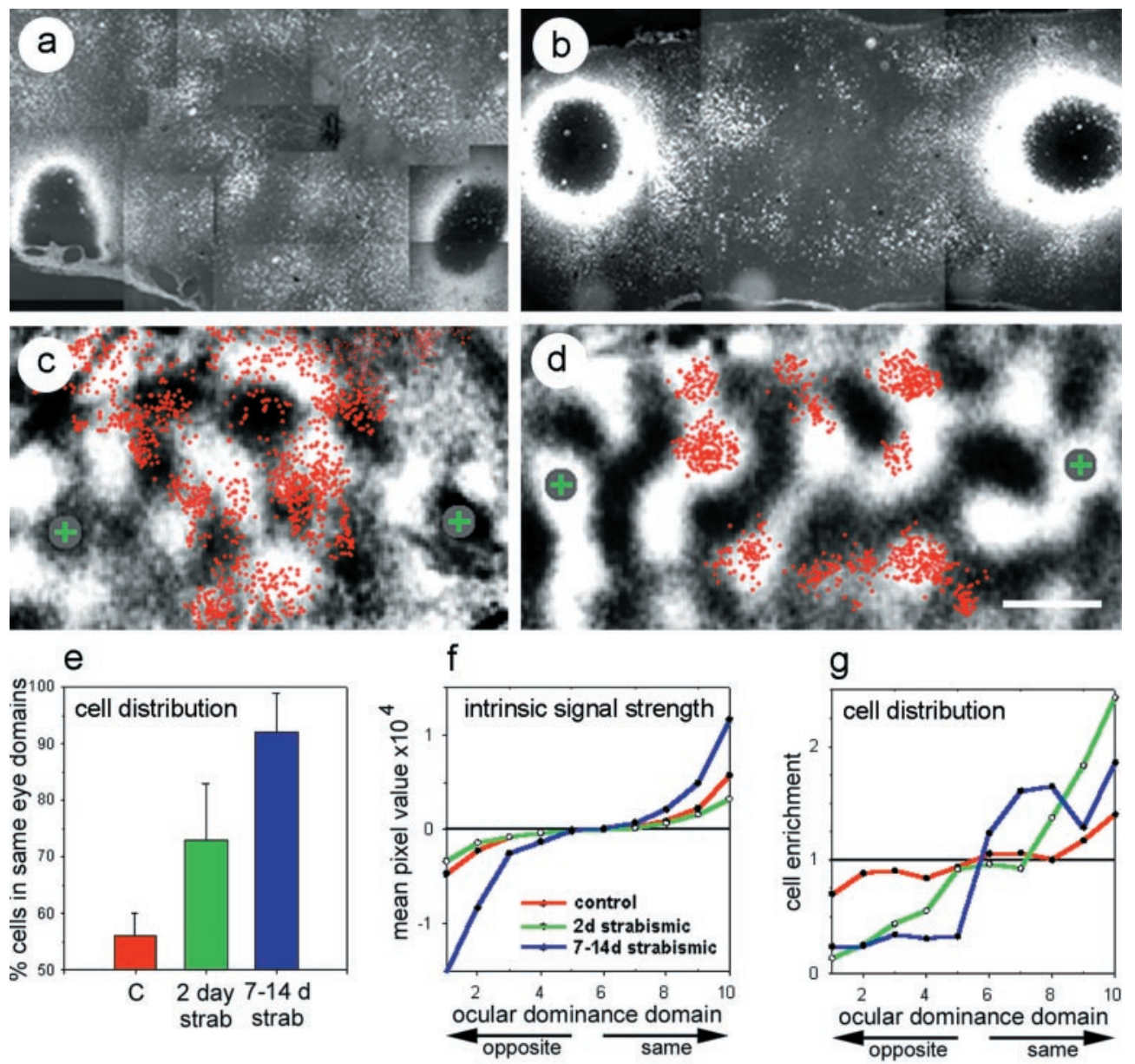

Figure 2. Ocular dominance distribution of retrogradely labeled cells. Anatomical sections showing the distribution of cells retrogradely labeled with WAHG in a normally sighted $(a)$ and a $2 \mathrm{~d}$ strabismic $(b)$ kitten. The large black circles surrounded by white halos on the left and right sides of each figure are the silver-enhanced injection sites. In sections that had not been silver-intensified, the bulk of the injection was restricted to an area of between 100 and $200 \mu \mathrm{m}$, which is much smaller than the region of dark precipitate present after silver intensification. The bright granular labeling between the two injection sites are gold-labeled cells. In these two examples, two injections were made into each hemisphere. $c$ and $d$ show the distribution of the labeled cells from the sections in $a$ and $b$ (red dots) overlaid on optical maps of ocular dominance. It should be noted that $a$ and $b$ are photomontages of microscope fields that have been scaled and rotated by eye to approximate the computer-assisted alignment of the digitally plotted cells in $c$ and $d$. In $c$, the injections, each marked by a gray circle enclosing a green +, were targeted to two black regions of the ocular dominance map. In $d$, the injections were targeted to white regions of the ocular dominance map. Scale bar: $a-d, 1 \mathrm{~mm}$. A quantitative distribution of labeled cells $>1 \mathrm{~mm}$ from the injection site is shown in $e-g$. In $e$, the percentage of labeled cells that lay within ocular dominance domains dominated by the eye that dominates the injection site are graphed for normally sighted (red), $2 \mathrm{~d}$ strabismic (green), and 7-14 d strabismic (blue) kittens. This color scheme is maintained in $f$ and $g$ and in Figure $3 c-e$. Error bars in $e$ are SEM. In $f$ and $g$, the ocular dominance maps were divided into 10 zones of equal area. $f$ shows the difference in the strength of the intrinsic signal between the two eyes for the pixels in each of the 10 zones. On the $y$-axis, a value of 0 indicates that pixels were equally driven by each of the two eyes. Values near 1 or -1 indicate that pixels were dominated by the same eye that dominates the injection site, or the other eye, respectively. On the $x$-axis in $f$ and $g$, a value of 1 is a black region on the optical map, strongly dominated by the other eye. All injections were treated as if they were made into white regions of the ocular dominance map (10 on the $x$-axis). Values $6-10$, therefore, represent the half of the cortex that is dominated by the eye that dominates the injection site, with 6 being most binocular and 10 being most monocular. Values $1-5$, then, represent cortical domains dominated by the other eye. $g$ plots the fraction of labeled cells per unit area in each of the 10 ocular dominance zones. A value of 1 on the $y$-axis indicates no difference from a random distribution. Values $>1$ indicate an enrichment of labeled cells per unit area, whereas values $<1$ indicate an impoverishment of labeled cells relative to a random distribution.

Qualitatively, the long-range connections appear to be primarily eye-specific in both control and experimental animals, whereas little or no specificity is apparent in the connections closer than 1 $\mathrm{mm}$ in the control case (Fig. 3, compare $a, b$ ). When examined quantitatively, as with the WAHG-labeled cells above, rapid eye-specific segregation of horizontal connections was revealed. When the ocular dominance map was divided into halves favoring one eye or the other, $72 \pm 3 \%$ of labeled boutons located $>1 \mathrm{~mm}$ from the injection site in normally sighted kittens were found in the half of the cortex serving the same eye ( 3 hemispheres, 6 sections, 17,469 boutons) (Fig. 3c). Strabismus produced a rapid remodeling of long-range horizontal connections that increased their eye specificity. After $2 \mathrm{~d}$ of strabismic vision (4 hemispheres, 9 sections, 8356 boutons), $89 \pm 4 \%$ of boutons lay in the same-eye half of cortex, a significant increase compared with controls $(p<$ 0.05 ; two-tailed $t$ test). The remodeling of boutons after $2 \mathrm{~d}$ of strabismus appeared to be saturating, because extending the period of strabismus to 1-2 weeks (4 hemispheres, 9 sections, 10,682 boutons) produced no further increase in the overall eye specificity of connections ( $88 \pm 1 \%$ in same-eye half of cortex; different from control, $p<0.05)$.

As with our analysis of retrogradely labeled cells, we divided 

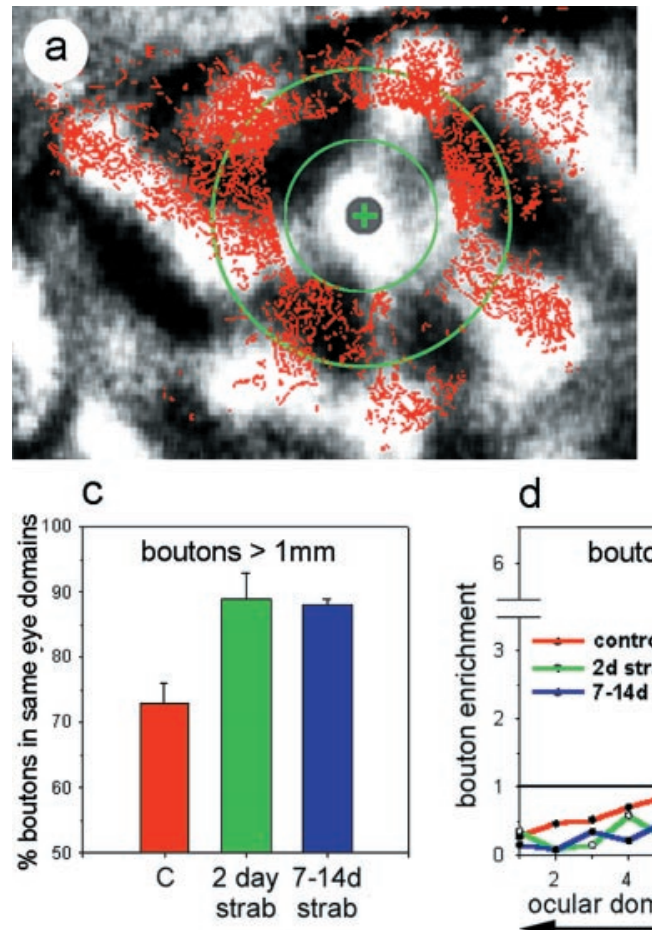

d

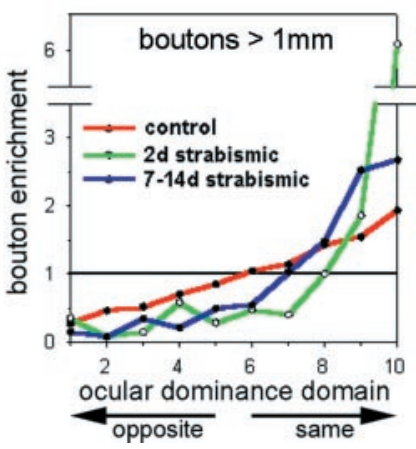

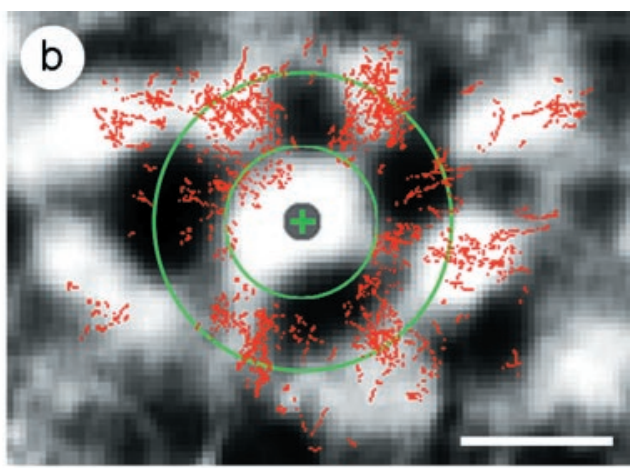

e

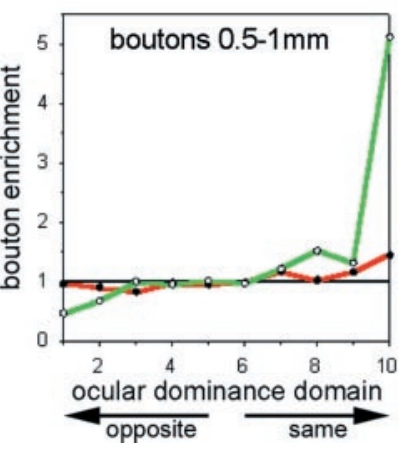

Figure 3. Ocular dominance distribution of anterogradely labeled boutons. $a$ and $b$ show the distribution of anterogradely labeled boutons ( red) overlaid on optical maps of ocular dominance (grayscale background) for a normally sighted $(a)$ and $2 \mathrm{~d}$ strabismic $(b)$ kitten. The injection site in each case is marked by a gray circle enclosing a green + . The inner green circle marks a $500 \mu \mathrm{m}$ radius from the injection site. The outer green circle marks a 1 mm radius from the injection site. Note that, in $a$, there are far more boutons present than in $b$, suggesting that a large fraction of the boutons may have been lost in this short time span. Scale bar (in $b$ ), $1 \mathrm{~mm}$. A quantitative distribution of boutons relative to ocular dominance domains is shown in $c$ and $d$ for boutons that lie $>1 \mathrm{~mm}$ from the injection site. As in Figure 2, control animals are shown in red, $2 \mathrm{~d}$ strabismic animals in green, and 7-14 $\mathrm{d}$ strabismic animals in blue. $c$ graphs the percentage of boutons that lie within the half of the cortical area dominated by the eye that dominates the injection site. Error bars represent SEM. $d$ plots the fraction of boutons per unit area in each of 10 ocular dominance zones. Colors are as in $c$, and ordinate and abscissa are as in Figure 2g. $e$ plots this fraction for boutons lying between $500 \mu \mathrm{m}$ and $1 \mathrm{~mm}$ from the injection site. Colors are as in $c$, and ordinate and abscissa are as in Figure $2 g$.

the optical maps of ocular dominance into 10 zones of equal area (see Materials and Methods for a detailed description of this analysis). Analyzing the eye specificity of anterogradely labeled connections in this manner, we see in the long-range connections of normally sighted animals a linear fall off in the density of projections to other cortical regions as a function of the ocular dominance of those regions (Fig. 3d). That is, the cells labeled by our injections into the peaks of ocular dominance columns of one eye extended their horizontal connections most densely into the peaks of other ocular dominance columns serving the same eye. The peaks of columns serving the other eye received the fewest connections, with a graded density of connections to the binocular regions in between. Such a pattern of projections would be expected to reflect the degree of correlated activity between the projection sites. Short-range connections (those to regions between 500 and $1000 \mu \mathrm{m}$ from the labeled cells) did not display any eye specificity in these control animals (Fig. 3e). Two days of strabismus dramatically reorganized this pattern of connections. The density of long-range connections to the very peaks of other ocular dominance columns serving the same eye was greater by a factor of 6 than would be expected from a uniform, random distribution of boutons (Fig. 3d). Connections to all of the ocular dominance zones of the other eye were dramatically impoverished by $\sim 75 \%$. Short-range connections in these animals exhibited similar specificity for the peaks of same-eye columns, but they were not impoverished in the $70 \%$ of the cortex lying between the extremes of ocular dominance (Fig. 3e).

\section{DISCUSSION}

These experiments demonstrate that experience can rapidly reorganize tangential connections within the upper layers of visual cortex at the height of the critical period. By some measures, these anatomical changes, like the physiological changes (Van Sluyters and Levitt, 1980; Levitt and Van Sluyters, 1982), are as great after $2 \mathrm{~d}$ as they are after 7-14 $\mathrm{d}$ of abnormal experience. Indeed, we do not know how fast the changes really are, for either the physiology or the anatomy, because both are so large by $2 \mathrm{~d}$. The time course is rapid enough that future studies should be able to investigate them by following individual axonal processes or dendritic spines in vivo (Lendvai et al., 2000) while such changes are occurring.

A second finding of these experiments is the linear relationship between cortical ocular dominance and the density of tangential connections in normal animals. Both this result and the reorganization after strabismus are exactly what would be predicted if the connections between two points in the cortex were established and maintained in proportion to the correlation between neuronal activity at the two points.

The eye specificity of tangential connections in normal animals is similar in magnitude to their orientation specificity revealed with similar techniques (Bosking et al., 1997; Kisvárday et al., 1997). These authors found that $\sim 60 \%$ of labeled boutons lay in columns whose preferred orientation was within $\pm 35^{\circ}$ of that of cells at the injection site. The degree of enrichment of connec- 
tions according to functional specificity in their work (1.54, calculated as $60 \%$ of connections confined to $38 \%$ or $70^{\circ} / 180^{\circ}$ of the cortical area by Bosking et al., 1997; this index was 1.62 according to Kisvárday et al., 1997) is almost identical to the eye specificity we find in normally sighted kittens (1.51 calculated from the four rightmost points of Fig. $3 d$, constituting $40 \%$ of the cortical area). It is also similar to, but less pronounced than, the segregation of long-range horizontal connections into specific monocular and binocular domains reported for primate V1 (Malach et al., 1993).

We cannot determine whether the plasticity seen in our experiments results from the rapid addition of new connections to same-eye zones or the loss of connections to zones dominated by the other eye. The analysis we used measures the fraction of labeled boutons or cells within each ocular dominance zone, divided by the fraction of cortical area in each zone over the regions covered by the boutons and cells and the gaps between them. Thus, it is not capable of distinguishing between an absolute increase in the connections to same-eye zones and an absolute decrease in connections to other zones, which would have the same effect on the fractional values. Although it seems likely that both addition and loss of connections take place (Silver and Stryker, 2000), resolving this question will require either timelapse imaging of identified processes or detailed measurements on large numbers of individual cells of known classes.

Although our findings from retrograde and anterograde labeling concur on the rapidity of plasticity, the quantitative differences between results from the two techniques suggest a model for the progressive rearrangement of tangential connections, as illustrated in Figure 4. Despite the fact that both sorts of injections were targeted to the peaks of ocular dominance columns, the retrograde tracer injections diff used more widely and labeled processes over a broader range of ocular dominance. The lesser enrichment of eye-specific connections seen with retrograde labeling is thus consistent with a more rapid and complete reorganization of connections to the peaks of ocular dominance columns than to the zones in between, which were binocularly driven at the beginning of the experiment.

Two other factors may contribute to the quantitative difference between results from the two techniques. The synaptic boutons that were counted presumably show sites of functional connections, whereas processes that pass through the injection site but do not make connections within it may take up retrograde label. In addition, retrograde labeling is a threshold phenomenon, and a cell might arborize extensively and with great specificity elsewhere but be labeled from a single naked process that extends through the injection site.

The difference seen with retrograde labeling between 2 and 7-14 d of strabismus is consistent with an ultimate binarization of the cortex into two regions, within each of which cells and connections become uniformly monocular. Within such a binary cortex, the greater diffusion of retrograde tracer is no longer an issue, because the diffusion is always less than the radius of an ocular dominance patch. The binary distribution of connections thus remains consistent with the structure of correlated activity in the cortex.

The rapid plasticity of tangential corticocortical connections stands in contrast to the slower reorganization of thalamocortical input. Two days of monocular deprivation (Movshon and Dursteler, 1977; Crair et al., 1997b) or strabismus (Van Sluyters and Levitt, 1980; Levitt and Van Sluyters, 1982) during the peak of the critical period causes a robust and saturating shift in the physiological organization of V1, but the anatomical substrate of

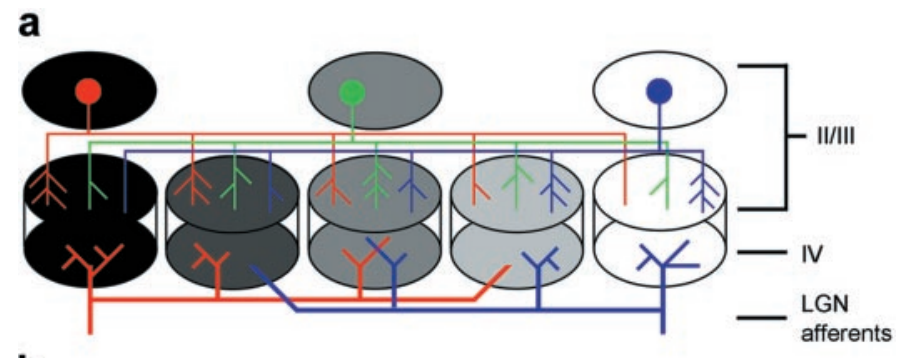

b

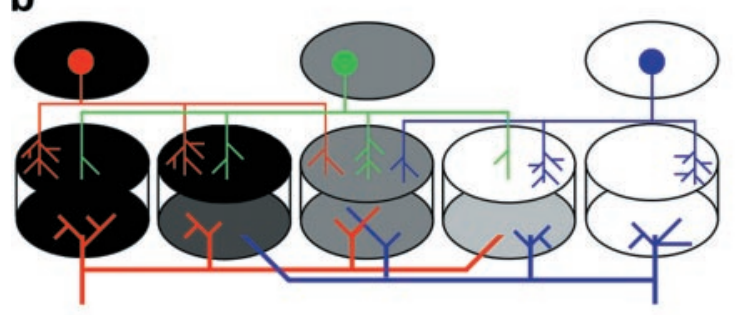

c
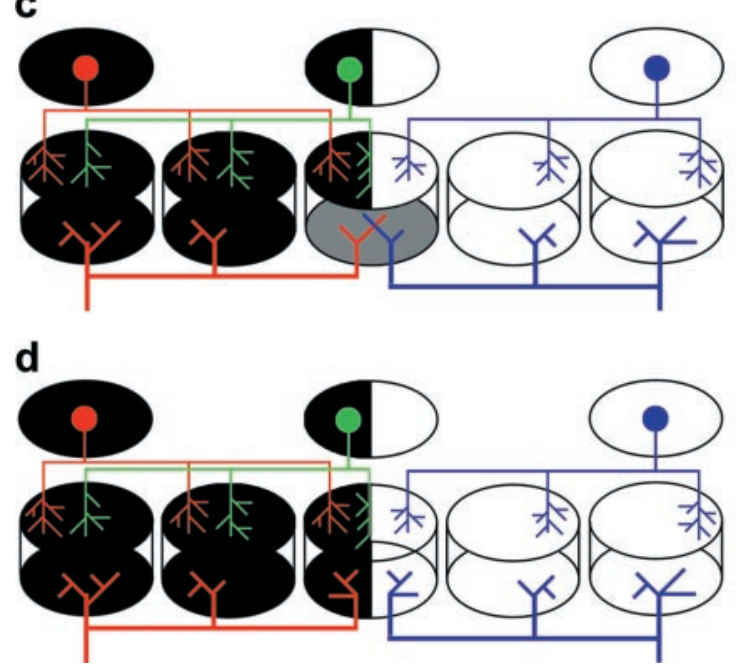

Figure 4. Schematic depiction of anatomical rearrangements underlying ocular dominance plasticity. In all panels, black domains are entirely dominated by one eye and white by the other eye, and the gray zones are equally responsive to both eyes. $a$, Normally sighted kitten. Layer II/III neurons at the peaks of ocular dominance (red and blue circles) extend horizontal connections preferentially to like-eye columns, with few connections extended to opposite-eye columns. Layer II/III neurons in binocular zones (green circle) extend connections preferentially to other binocular zones and to both ocular dominance peaks. $b$, After $2 \mathrm{~d}$ of strabismus, horizontal connections from ocular dominance extrema (layer II/III; red, blue) have completely withdrawn their connections to opposite-eye domains but retained their connections to the most binocular zone. Upper layer binocular neurons (green) have withdrawn approximately half of their connections to opposite eye domains. Changes in the ocular dominance of the upper layers are present, although no changes in the ocular dominance of layer IV are seen. No change in the lateral geniculate nucleus ( $L G N)$ afferents are present at this time (thick red and blue lines at the bottom of each panel). $c$, Four days after strabismus, pyramidal neurons in binocular zones have entirely withdrawn their horizontal connections to opposite-eye domains. Horizontal connections from ocular dominance extrema have sprouted new connections in likeeye domains. Remodeling of the upper layers is complete now. Lateral geniculate nucleus afferents to opposite-eye domains have only begun to withdraw, and there is still strong overlap in binocular zones. $d$, By 7-14 d after strabismus, the remodeling of the lateral geniculate nucleus afferents to layer IV is complete. Inappropriate connections to oppositeeye domains are entirely lost, as is the overlap of left- and right-eye afferents in the most binocular region of layer IV. 
this physiological change has remained enigmatic. Using Phaseolus lectin, Antonini and Stryker $(1993,1996)$ measured the lengths, numbers of branch points, and the densities of terminal arborizations of geniculocortical afferents in kittens after 4, 7, and $28 \mathrm{~d}$ of deprivation. Silver and Stryker (1999) then measured the density of presynaptic terminals and synaptic vesicle proteins after 2 and $7 \mathrm{~d}$ of MD. Together, their data support a model in which retraction of deprived geniculocortical afferents has not yet begun by $2 \mathrm{~d}$ after the onset of deprivation (a time when physiological remodeling is effectively complete), is slight after $4 \mathrm{~d}$, and is complete only after $7 \mathrm{~d}$. Changes in geniculocortical afferents thus appear to be too slow to account for the rapid physiological changes in ocular dominance. The slower reorganization of thalamocortical connections may, however, be essential for consolidating the ocular dominance changes that are first apparent in the physiology and the tangential connections.

The more rapid changes in ocular dominance in the upper layers than in layer IV of cortex (Trachtenberg et al., 2000) might result from changes in ascending inputs from IV to II/III (Feldman, 2000) or from changes in horizontal connections within the upper layers (Cynader, 2000). The present findings establish the reality of the latter suggestion, but they do not rule out additional contributions from the former.

The remodeling of tangential connections found here is the first anatomical change shown to be rapid enough to serve as the substrate of physiological changes in developing visual cortex.

\section{REFERENCES}

Antonini A, Stryker MP (1993) Rapid remodeling of axonal arbors in the visual cortex. Science 260:1819-1821.

Antonini A, Stryker MP (1996) Plasiticity of geniculocortical afferents following brief or prolonged monocular occlusion in the cat. J Comp Neurol 369:64-82.

Basbaum AI Menterey D (1986) Wheat germ agglutinin-aPoHRP gold: a new retrograde tracer for light- and electron-microscopic single- and double-label studies. J Comp Neurol 13:3549-3573.

Bosking WH, Zhang Y, Schofield B, Fitzpatrick D (1997) Orientation selectivity and the arrangement of horizontal connections in tree shrew striate cortex. J Neurosci 17:2112-2127.

Buonomano DV, Merzenich MM (1998) Cortical plasticity: from synapses to maps. Annu Rev Neurosci 21:149-186.

Crair MC, Ruthazer ES, Gillespie DG, Stryker MP (1997a) Ocular dominance peaks at pinwheel center singularities of the orientation map in cat visual cortex. J Neurophysiol 77:3381-3385.

Crair MC, Ruthazer ES, Gillespie DG, Stryker MP (1997b) Relationship between ocular dominance columns and pinwheel center singularities of the orientation map in visual cortex of monocularly deprived cats. Neuron 19:307-318.

Cynader M (2000) Strengthening visual connections. Science 287: 1943-1944.
Feldman D (2000) Timing-based LTP and LTD at vertical inputs to layer II/III pyramidal cells in rat barrel cortex. Neuron 27:45-56.

Gilbert CD (1998) Adult cortical dynamics. Physiol Rev 78:467-485.

Gilbert CD, Wiesel TN (1989) Columnar specificity of intrinsic horizontal and corticocortical connections in cat visual cortex. J Neurosci 9:2432-2442.

Hess G, Aizenman CD, Donoghue JP (1996) Conditions for the induction of long-term potentiation in layer II/III horizontal connections of the rat motor cortex. J Neurophysiol 75:1765-1778.

Hubel DH, Wiesel TN (1963) Single-cell responses in striate cortex of kittens deprived of vision in one eye. J Neurophysiol 26:1003-1017.

Hubel DH, Wiesel TN (1965) Binocular interaction in striate cortex of kittens reared with artificial squint. J Neurophysiol 28:1041-1059.

Issa NP, Trachtenberg JT, Chapman B, Zahs K, Stryker MP (1999) The critical period for ocular dominance plasticity in the ferret's visual cortex. J Neurosci 19:6965-6978.

Kisvárday ZF, Tóth E, Rausch M, Eysel UT (1997) Orientation-specific relationship between populations of excitatory and inhibitory lateral connections in the visual cortex of the cat. Cereb Cortex 7:605-618.

Lendvai B, Stern EA, Chen B, Svoboda K (2000) Experience-dependent plasticity of dendritic spines in the developing rat barrel cortex in vivo. Nature 404:876-881.

Levitt FB, Van Sluyters RC (1982) The sensitive period for strabismus in the kitten. Dev Brain Res 3:323-327.

Lowel S, Singer W (1992) Selection of intrinsic horizontal connections in the visual cortex by correlated neuronal activity. Science 255:209-212.

Malach R, Amir Y, Harel M, Grinvald A (1993) Relationship between intrinsic connections and functional architecture revealed by optical imaging and in vivo targeted biocytin Injections in primate striate cortex. Proc Natl Acad Sci USA 90:10469-10473.

Movshon JA, Dursteler MR (1977) Effects of brief periods of unilateral eye closure on the kitten's visual system. J Neurophysiol 40:1255-1265.

Olson CR, Freeman RD (1975) Progressive changes in kitten striate cortex during monocular vision. J Neurophysiol 38:26-32.

Rioult-Pedotti MS, Friedman D, Hess G, Donoghue JP (1998) Strengthening of horizontal cortical connections following skill learning. Nat Neurosci 1:230-234

Ruthazer ES, Stryker MP (1996) The role of activity in the development of long-range horizontal connections in area 17 of the ferret. J Neurosci 16:7253-7269.

Sanes JN, Donoghue JP (2000) Plasticity and primary motor cortex. Annu Rev Neurosci 23:393-415.

Shatz CJ, Stryker MP (1978) Ocular dominance in layer IV of the cat's visual cortex and the effects of monocular deprivation. J Physiol (Lond) 281:267-283.

Silver MA, Stryker MP (1999) Synaptic density in geniculocortical afferents remains constant after monocular deprivation in the cat. J Neurosci 19:10829-10842.

Silver MA, Stryker MP (2000) Distributions of synaptic vesicle proteins and GAD65 in deprived and nondeprived ocular dominance columns in layer IV of kitten visual cortex are unaffected by monocular deprivation. J Comp Neurol 422:652-664.

Trachtenberg JT, Trepel C, Stryker MP (2000) Rapid extragranular plasticity in the absence of thalamocortical plasticity in the developing primary visual cortex. Science 287:2029-2032.

Van Sluyters RC, Levitt FB (1980) Experimental strabismus in the kitten. J Neurophysiol 43:686-699.

Wallace H, Fox K (1999) Local cortical interactions determine the form of cortical plasticity. J Neurobiol 41:58-63. 